\title{
Adenoid Cystic Carcinoma of Buccal Mucosa
}

\author{
Sanjay Singh • Gokkulakrishnan · Jinendra Jain • \\ Sachin Pathak $\cdot$ Kumar Tathagat Singh
}

Received: 14 January 2010/Accepted: 24 April 2010/Published online: 25 November 2010

(C) Association of Oral and Maxillofacial Surgeons of India 2010

\begin{abstract}
Adenoid cystic carcinoma is a malignant neoplasm most commonly originating in salivary glands of head and neck region. Here we present a case report of a 50 year old male who was diagnosed with adenoid cystic carcinoma of the right buccal mucosa. The peculiarity of the lesion and the approach we made is the key factor in the presentation.
\end{abstract}

Keywords Carcinoma - Adenoid cystic - Malignant neoplasm $\cdot$ Salivary gland disorder

\section{Introduction}

Adenoid cystic carcinoma was first described by Theodor Bilroth in 1856, as cylindromas, in his histological studies where he described it as long amorphous compartments called "cylinders". It is only recently that the tumor has been renamed as adenoid cystic carcinoma (ACC) (Kaiser), which is a rare tumor accounting for less than $1 \%$ of all head and neck malignancies and $10 \%$ of all salivary gland neoplasms [1]. The tumor extends well beyond the visible and palpable limits of the salivary gland region; this infiltrative capacity is the hallmark of this tumor. Pain and perineural spread along nerve sheath is usually noted [2]. Majority of the tumors arise in the major salivary glands, minor salivary glands of the oral cavity and mucous glands of upper respiratory tract. Other primary sites are breast, lacrimal glands, lung and prostate. Lymph node metastases

S. Singh $(\varangle) \cdot$ Gokkulakrishnan · J. Jain - S. Pathak ·

K. T. Singh

Department of OMFS, Kothiwal Dental College \& Research

Centre, Moradabad, India

e-mail: Prof.SanjaySingh@gmail.com are unusual; hematogenous spread, often to the lungs is quite characteristic [3], metastasis to kidney being extremely rare [4]. ACC is known for its prolonged clinical course, multiple recurrence rates and delayed onset of distant metastasis.

\section{Case Presentation}

A 50 year-old male reported with a chief complaint of swelling in the right buccal vestibule with mild pain since one and a half year. A year ago, no specific treatment was sought for the same. Extra orally there was facial asymmetry with swelling seen on the right side of the face on the cheek (Fig. 1). Facial nerve function was normal. Intraoral examination revealed a red, firm lesion of the right buccal mucosa measuring $3 \mathrm{~cm}$ in greatest dimension (Fig. 2). Patient was dentulous and there was mild pain associated with the lesion. Incisional biopsy was performed at our center which revealed cystic spaces filled with eosinophilic coagulum and arrangement of tumor cells predominantly in cribriform pattern and in anastamosing thin strands with hyalinised intervening stroma. Overall histopathological picture was suggestive of ACC (Fig. 3). A second incisional biopsy was performed elsewhere which confirmed the same histopathological diagnosis. Adenoid cystic carcinoma is considered to invade adjacent tissues by perineural route and metastasize by hematogenous route. The common sites for metastasis are lungs and deep organs like liver, spleen and kidney. To rule out any metastasis; ultrasound of upper abdomen, PA view of chest, Non contrast proton MRI of the oral cavity, The axial CT scan of head and face, submentovertex view of skull $\left(35^{\circ}\right)$, lateral neck radiograph, PA view of pelvic girdle, CT scan of chest, were performed. The ultrasound of upper 
abdomen showed normal liver, gall bladder, pancreas, spleen and kidneys with no evidence of any metastatic mass. PA view of chest showed no active lung parenchymal disease non contrast proton MRI of the oral cavity was suggestive of a well defined mass in the masticator space on right side extending up to postero-lateral wall of maxillary sinus anteriorly and ramus of mandible and medial pterygoid muscle posteriorly. The axial CT scan of head and face was suggestive of space occupying lesion on the right cheek involving the masticator space (Fig. 4). Submentovertex view of skull $\left(35^{\circ}\right)$, lateral neck radiograph, PA view of pelvic girdle, CT scan of chest were done which confirmed negative metastasis of the tumor. After confirming the nature of lesion and evidence of no distant organ metastasis, the patient was posted for surgery in our center under general anaesthesia. A curvilinear incision starting from ascending border of ramus till the vestibule adjacent to right upper six region was made (Fig. 5). After the fibres of buccinators were dissected, demarcation of the lesion was noticed and it was excised in toto (Fig. 6). Post operative recovery of the patient was uneventful. The final diagnosis of the surgical specimen was adenoid cystic carcinoma. Post operative necessary clinical and radiological investigations were performed which were within normal limits (Fig. 7). The patient is being followed up till date (since 3 years) and is still free of disease.

\section{Discussion}

Adenoid cystic carcinoma is a malignancy of salivary gland origin. It clinically presents as a small, slowly growing lesion but with extensive subclinical invasion and a possibility of early metastasis. It is a malignancy that

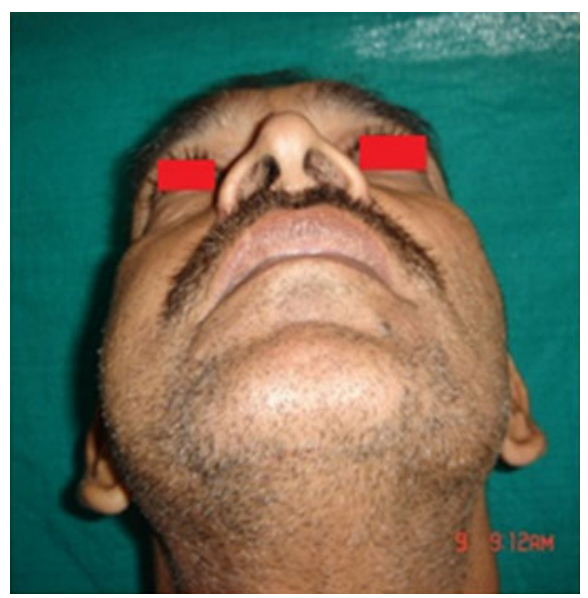

Fig. 1 Pre operative picture, notice the swelling on right side of the face with facial asymmetry

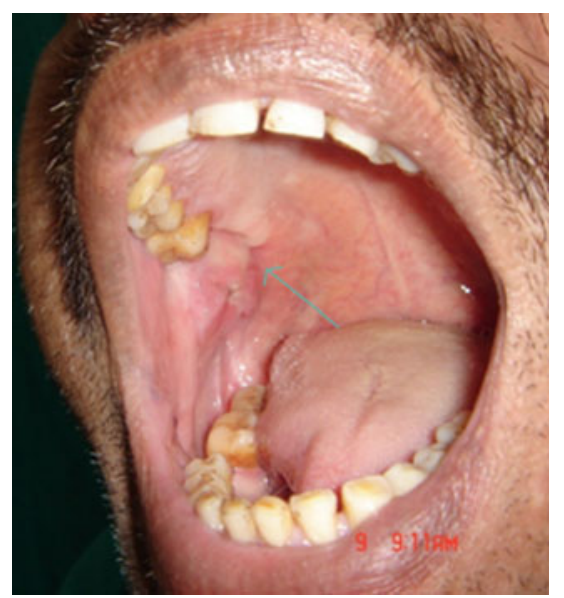

Fig. 2 Intra oral view arrow pointing the lesion

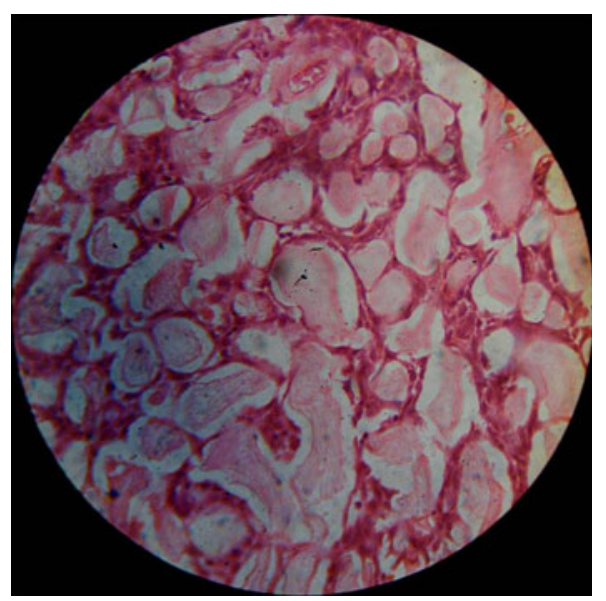

Fig. 3 Histopathological picture (H\&E stain, $40 \times$ magnification)

reinforces the point that tumor growth rate and metastatic capabilities are independent tumor properties [5]. Although the buccal mucosa is an uncommon site for adenoid cystic carcinoma, the relatively indolent growth pattern of this case and its location is rather atypical for this type of salivary gland malignancy. ACC has a peak incidence in the fourth to sixth decades of life with and equal male to female distribution, which was similar to our case. Histologically, our case had a classical cribriform pattern of cells which according to many authors has a favorable prognosis as compared to solid pattern [6]. Adenoid cystic carcinomas of the minor glands have been reported to have a worse prognosis than those of the major salivary glands. This may be explained by the fact that tumors of the minor salivary glands can more readily infiltrate extra-glandular soft tissues and bone, thereby allowing for increased dissemination of the tumor. We performed a thorough microscopic evaluation of the specimen for marginal clearance and scans of patient to check for any recurrence of the tumor. The treatment aspect of the tumor is chiefly 


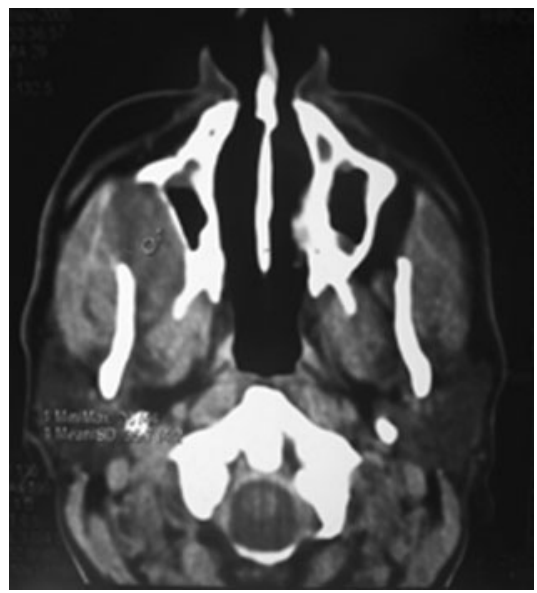

Fig. 4 CT revealing the tumor extent

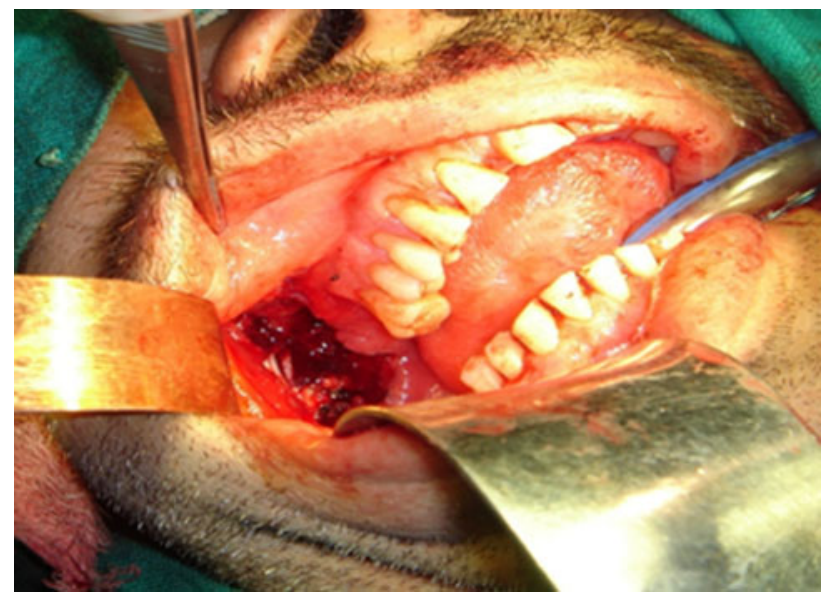

Fig. 5 Incision and exposure

surgical although in some cases surgery has been successfully coupled with radiotherapy. Radiation therapy as the sole treatment modality seems to be inadequate for ACC. Unlike most other type of salivary gland carcinoma, distant metastasis is far more frequent in ACC (as lungs, liver, kidneys, pelvic girdle.) Lymphatic spread is far less common in this tumor. In our patient we preferred surgical excision as the sole treatment because histopathologically the lesion was cribriform type which has better prognosis than the other variants, after surgical excision of the tumor, the margins of the tumor were clear microscopically and regular follow up of the patient with repeated scans and radiographs has nor revealed any signs of recurrence or metastasis till date. There is limited role of localized radiotherapy due to characteristic distant metastasis. The underlying principles in all ACC therapy are that tumor cells extend well beyond the clinical or radiographic

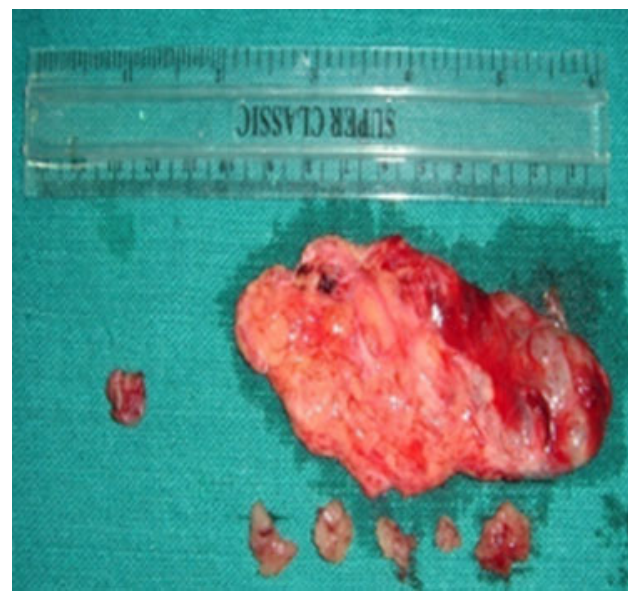

Fig. 6 Excised specimen

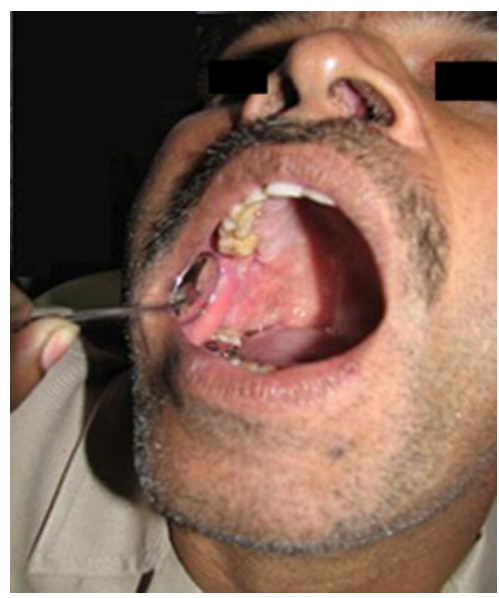

Fig. 7 Postoperative photograph

margins and that this tumor undergoes not only perineural invasion but perineural spread. Therefore, it generally requires excision with the widest margins possible. The overall prognosis relates to several factors. Histologically, solid patterns bode worse than cribriform patterns, and in our case it was of cribriform type [7]. Clinical size greater than $4 \mathrm{~cm}$ indicates an even greater subclinical spread and, therefore, is associated with a worse prognosis. Delayed diagnosis and/or delayed treatment also worsen the prognosis, which was true in our case as we made an early diagnosis with a prompt treatment. Surgical margins that are not clear or are 'close' despite postoperative radiotherapy are also associated with a worse prognosis; this is the single most important factor associated with local recurrence, whereas in our patient, the borders of the surgical specimen was free of the disease indicating a better prognosis. 


\section{Conclusion}

Adenoid cystic carcinomas have a variable prognosis. The 5 year survival rate is $75 \%$, but 10 year survival rate is only $20 \%$, and survival at 15 years is about $10 \%$ [5]. Other factors which adversely affect the prognosis are perineural spread, distant metastasis and recurrent local lesions. We preferred surgical excision as the sole treatment for this patient because pre operatively there was no evidence of any distant metastasis [8]; histopathologically the lesion was cribriform type which has better prognosis than the other variants. Histopathological examination revealed that the margins were free of the disease. Adenoid cystic carcinoma usually does not metastasizes to lymph nodes, distant metastasis into the lungs via blood-borne routes has been suggested [5]. However in our case metastasis was not found and the patient is being followed up at regular intervals and is free of disease and metastasis till date (3years postoperatively).

\section{References}

1. Dutta NN, Baruah R, Das L (2002) Adenoid cystic carcinomaclinical presentation and cytological diagnosis. Indian J Otolaryngol Head Neck Surg 54(1):62-64
2. Sternberg SS (1997) Tumours of salivary gland. In: Diagnostic surgical pathology. 3rd edn. Lippincott Williams \& Wilkins, Philadelphia, p. 867

3. Phanindra Kumar V et al (2003) Adenoid cystic carcinoma of nasal cavity - a case report. Indian J Otolaryngol Head Neck Surg 55(1):43-46

4. Srivastava S, Jaiswal R, Agarwal A, Singh PK, Singh SN (2007) Cytological diagnosis of adenoid cystic carcinoma of the parotid metastatic to kidney and lung. J Cytol 24(4):201-202

5. Robert E. Marks, Diane Stern (2003) Salivary gland neoplasm. In: Oral and maxillofacial pathology: a rationale for diagnosis and treatment. 1st edn. Quintessence books, New Malden, pp 550-553

6. Robert A Ord (2008) Salivary gland disease. In: Raymond J Fonseca (ed) Oral and maxillofacial surgery, 1st edn, vol 5 (surgical pathology). W.B Saunders Company, Philadelphia, pp 290-291

7. Chomette $G$ et al (1982) Adenoid cystic carcinoma of minor salivary glands. Analysis of 86 cases. Clinico-pathological, histoenzymological and ultrastructural studies. Virchowa Arch (Pathol Anat) 395(5):289-301

8. Luna-Oritz Kuanhyama et al (2009) Adenoid cystic carcinoma of the tongue- clinicopathological study and survival analysis. Head Neck Oncol 1:15. doi:10.1186/1758-3284-1-15 